\title{
Posttraumatic knee osteoarthritis following anterior cruciate ligament injury: Potential biochemical mediators of degenerative alteration and specific biochemical markers (Review)
}

\author{
HONG LI $^{1}, \mathrm{CHEN} \mathrm{CHEN}^{2}$ and SHIYI CHEN ${ }^{1}$ \\ ${ }^{1}$ Department of Sports Medicine, Huashan Hospital, Shanghai 200040; ${ }^{2}$ Department of Orthopaedics, \\ Zhongshan Hospital, Shanghai 200032, P.R. China
}

Received February 10, 2014; Accepted November 5, 2014

DOI: $10.3892 /$ br. 2014.404

\begin{abstract}
As a common injury, anterior cruciate ligament (ACL) injury is unable to heal itself naturally, which possibly increases knee instability, accelerates the risk of joint degeneration and leads to knee osteoarthritis (OA) in the ACL-injured knee. Thus, ACL reconstruction using an autograft or allograft tendon is proposed to maintain the biomechanical stability of the knee joint. However, previous studies demonstrate that surgical management of ACL reconstruction failed to abrogate the development of OA completely, indicating that biochemical disturbance is responsible for the osteoarthritic changes observed following ACL injury. Inflammatory mediators are elevated subsequent to ACL injury or rupture, inducing matrix metalloproteinase production, proteoglycan degradation, collagen destruction, chondrocyte necrosis and lubricin loss. These potential biochemical mediators may aid in the development of effective biological management to reduce the onset of future posttraumatic OA. Furthermore, during the degenerative process of cartilage, there are a number of cartilage-specific biomarkers, which play a critical step in the loss of structural and functional integrity of cartilage. The present review illustrates several specific biomarkers in the ACL-injured knee joint, which may provide effective diagnostic and prognostic tools for investigating cartilage degenerative progression and future posttraumatic OA of ACL-injured patients.
\end{abstract}

\section{Contents}

1. Introduction

2. Degenerative alteration of knee cartilage: Matrix metalloproteinase production

Correspondence to: Professor Shiyi Chen, Department of Sports Medicine, Huashan Hospital, 12 Wulumuqi Zhong Road, Shanghai 200040, P.R. China

E-mail: sychen11@163.com

Key words: osteoarthritis, anterior cruciate ligament, cartilage, knee, inflammatory mediators, biomarker
3. Proteoglycan degradation

4. Collagen destruction

5. Chondrocyte necrosis

6. Biomarkers of cartilage degeneration

7. Conclusions and perspectives

\section{Introduction}

Anterior cruciate ligament (ACL) rupture is one of the most common injuries in sports activity, often accompanied by articular cartilage damage and meniscal tear (1-3). ACL deficiency can significantly alter the knee joint biomechanics $(4,5)$, which will subsequently result in secondary damage to the cartilage with increasing time from injury (6-9). As a consequence, the articular cartilage of load-bearing joints is inexorably destroyed and the cartilage degeneration develops in an accelerated rate in the ACL-deficient knee (10-12). With regards to this, ACL reconstruction with an autograft or allograft tendon becomes a major method in maintaining the stability and normal biomechanics of the knee joint.

Restoring knee joint stability, however, was unable to decrease the incidence of posttraumatic osteoarthritis (OA) following ACL reconstruction, as previous studies have indicated that cartilage degeneration or radiographic signs of OA were detected in patients with ACL-reconstructed knees several years postoperatively (13-15). In a recent study examining 210 ACL-reconstructed patients, it was stated that $71 \%$ of the patients had radiographic knee OA and $24 \%$ showed moderate or severe radiographic knee OA postoperatively (16). Collectively, these data indicate that biomechanical instability is not the only cause for the occurrence of OA in the knee joint with ACL injury. Administered within the first month following ACL tear, intra-articular interleukin (IL)-1 receptor antagonist reduced knee pain and improved function over a 2-week interval (17). In addition to biomechanical disturbance, various biochemical alterations caused by ACL injury, such as inflammatory cytokines infiltration, matrix metalloproteinase (MMP) production, proteoglycan (PG) degradation, collagen destruction, chondrocyte death and lubricin loss, are postulated to play an important role in the development of posttraumatic OA. 


\section{Degenerative alteration of knee cartilage: MMP produc- tion}

In the ACL-injured knee, synoviocytes, chondrocytes and other intra-articular tissues are activated to produce several inflammatory mediators, such as IL-1 $1 \beta$, IL-6, IL-8, IL-10 and tumor necrosis factor- $\alpha$ (TNF- $\alpha)(18,19)$. IL-1 modulates the metabolic balance of cartilage in dual means, decreasing the production of the cartilage matrix components and promoting the expression of MMPs, including MMP-1, MMP-3, MMP-9, MMP-10 and MMP-13 (20-23). Recruitment of IL-1 to the IL-1 receptor activates four intracellular signaling pathways, including three mitogen-activated protein kinases (MAPKs) pathways [the extracellular signal-regulated kinase (Erk), p38 MAPK, c-Jun N-terminal kinases (JNK)] and the nuclear factor- $\kappa \mathrm{B}(\mathrm{NF}-\kappa \mathrm{B})$ pathway. Activation of transcription factors, such as NF- $\mathrm{kB}$ (activated by Erk and p38) and activator protein-1 (activated by JNK), induces the expression of numerous MMPs (24). Thus far, 24 associated products of the MMP family have been found in mammals (25). Secreted and anchored to the cell membrane, MMPs catalytically interact with various proteins, including protein components of the extracellular matrix. In a load-induced injury model on the mature bovine cartilage, Lin et al (26) found that the expression of MMP-3 increased consistently in the superficial-loading cartilage zone followed by cell death. MMP-3 can activate procollagenase, which induces the degradation of cartilage matrix, consequently affecting the cartilage repair. Thus, the MMP-3 selective inhibitor may be an effective option for retarding such osteoarthritic changes (27).

\section{PGs degradation}

PG contains a protein core with glycosaminoglycans (GAGs) side chains. As a main component, GAG is one important specific PG biomarker. In the ACL-injured knee, infiltration of numerous inflammatory cytokines accelerates the degradation of PGs and the decrease of GAGs in the cartilage to a significant extent (28). In an ACL-injured rabbit model (29), loss of GAGs was observed in the cartilage layer for a long time period. Hattori et al (29) found that the average thickness of the GAG-stained area in the ACL-injured group $(7.7 \mu \mathrm{m})$ was significantly less compared to the control group $(69.4 \mu \mathrm{m})$, even six weeks after surgery. The apoptosis of chondrocytes has been proposed to induce GAG loss. In a similar ACL-injured rabbit model, it was also proved that apoptosis of chondrocytes lead to the decrease of GAGs in the cartilage layer of the ACL insertion following resection (30).

MMPs and aggrecanases cleave the GAGs of aggrecan at specific peptide bonds and release fragments. The proteolytic cleavage process has a destructive effect, resulting in the loss of GAGs from the matrix (31). MMPs have been reported as responsible for the $\mathrm{C}$-terminal catabolic processing of aggrecan, which may accelerate the loss of matrix aggrecan in the articular cartilage (32). Cleavage of aggrecan in the interglobular domain between the N-terminal G1 and G2 globular domains is considered of greatest pathological importance, as this releases the GAG-bearing region of aggrecan from the cartilage matrix (33). Furthermore, the study by Caterson et al (34) showed that aggrecanase is primarily responsible for the catabolism and loss of aggrecan from the articular cartilage in the early stages of arthritic joint diseases, and MMPs begin to mediate the degradation of the small proportion of aggrecan remaining in the tissue with the occurrence of collagen catabolism at later stages. The cleavage of the Asn341-Phe342 bond in the interglobular domain can be attributed to the proteolytic activity of MMPs, while aggrecanase is responsible for the cleavage of the Glu373-Ala374 bond in the interglobular domain. In a previous study, Sondergaard et al (35) found that inhibition of the activation of MAPK p38, p44/42 and Src family prevented the degradation of proteolytic cartilage by blocking MMP synthesis and activity and MAPK p44/42 was essential for the degradation of aggrecan mediated by aggrecanase. Understanding of these detailed signaling pathways involved in the matrix degrading process may aid in the potential usage of corresponding inhibitors to treat the damaged cartilage.

\section{Collagen destruction}

Collagen, as an important structural macromolecule of cartilage, contributes to $\sim 60 \%$ of the dry weight of articular cartilage and the type II collagen accounts for $90-95 \%$ of the articular cartilage collagen. In the early stage following ACL rupture, expression of the type II and type I collagens is elevated temporarily in the articular cartilage (36). However, with the consistent infiltration of inflammatory cytokines and activation of catabolic proteinases, cartilage specific macromolecules, such as type II collagen, are destroyed. Different from PGs, collagen loss is believed to be irreversible. Subsequently, the full thickness cartilage lesion fail to heal itself with biological repair by type II collagen, rather, they become filled with a fibrocartilage repair tissue that is deficient of the biomechanical properties. IL-1 reduces the production of type II collagen by modulating the expression of transcription factors Sp1 and Sp3 and it can also downregulate the expression of transforming growth factor- $\beta$ (TGF- $\beta$ ) type II receptor, which has a significant influence on the synthesis of type II collagen (37). In the study by Chadjichristos et al (38), it was found that the production of newly synthesized collagens is inhibited by IL-1 $\beta$ in proliferating rabbit articular chondrocytes, accompanied by a decreased level of steady-state type II collagen. IL- $1 \beta$ downregulates the transcription of the COL2Al gene through a $-41 /-33$ basepair sequence that is bound by a multimeric complex, including $\mathrm{Sp} 1$ and $\mathrm{Sp} 3$ transcription factors. Furthermore, Porée et al (39) found that IL-6 and/or soluble IL-6 receptor (sIL-6R) inhibits the production of type II collagen in rabbit articular chondrocytes at the transcriptional level. IL-6, sIL-6R or a combination can reduce the $\mathrm{Sp} 1 / \mathrm{Sp} 3$ ratio, as well as DNA-binding activities, thus inhibiting COL2Al transcription.

\section{Chondrocyte necrosis}

Several reasons were considered to be responsible for chondrocyte necrosis. One is the pullout force of ACL insertion. Previously, it was reported that $\sim 42 \%$ of chondrocyte apoptosis and degenerative histological changes have been observed in human ACL tibial insertion cartilage from days 19 to 206 after ACL rupture (40). Due to an imbalance between cell death and 
cell proliferation in the ACL insertions following ACL rupture, the decrease of chondrocytes may lead to histological changes of the cartilage layer in the insertions (41). Another reason is the large external force that results in bone marrow edema lesions (BMELs) (42). Bone marrow necrosis and fibrosis appeared in the BMELs (43). The cartilage overlying BMELs sustains irreversible injury and cartilage degeneration lasts with increasing chondrocyte necrosis even subsequent to ACL reconstruction (44). Furthermore, the abnormal biomechanics of the injured knee also influenced cartilage morphology, and posttraumatic and new bone marrow lesions (45). Articular cartilage overlying the bone bruise lesions showed chondrocytes necrosis and PGs loss (46-48).

\section{Biomarkers of cartilage degeneration}

ACL injury is believed to accelerate the development of knee OA. As mentioned previously, inflammatory factors continue to influence and damage the knee cartilage matrix, although the biomechanical stability recovers following ACL reconstruction. In order to effectively prevent and abrogate the degenerative alteration of knee cartilage, there is an increasing interest to improve the understanding of the early biological changes subsequent to ACL injury and at the follow-up visit for reconstructive surgery by investigating specific biomarkers in synovial fluid, serum or urine. Catterall et al (28) examined 21 biomarkers in serum and synovial fluid samples (including 20 in synovial fluid and 13 in serum, with 12 biomarkers measured in the two fluids). These biomarkers can be divided into PG, collagen and synovial fluid biomarkers. With the significant release of GAGs into the synovial fluid, there would be a large amount of GAGs detected in the synovial fluid (28). GAGs in synovial fluid can be one specific biomarker providing effective diagnostic information for cartilage PG turnover in the ACL-injured knee $(49,50)$.

Within the early time following ACL injury, the collagen-related biomarkers can be detected in synovial fluid. The progression of knee OA is associated with alterations in the systemic levels of the type II collagen metabolism biological markers (51). In general, collagen biomarkers contain C-terminal cross-linked telopeptide type II collagen (CTX-II), C-terminal cross-linked telopeptide type I collagen and N-terminal telopeptides of type I collagen. Presently, there is an increasing interest in monitoring the CTX-II level for OA progression, as high levels of the cartilage turnover biomarker CTX-II predict an increased risk of radiological progression (52-54). Lohmander et al (52) reported the release of CTX-II in synovial fluid from patients with ACL injury. The concentrations of CTX-II in synovial fluid were much higher in patients with ACL injury compared to the healthy control group, and the mean levels of CTX-II in synovial fluid were higher compared to the healthy levels at all time intervals following joint injury.

In synovial fluid, lubricin is believed to be one specific biomarker. Lubricin is expressed by the proteoglycan 4 gene and synthesized in a number of tissues, including cartilage, meniscus and tendon (55-57). As a disease-regulating cytoprotective glycoprotein, it plays an important role in the protection of articular cartilage structure, maintenance of articular low-friction and prevention of joint OA (58-61). A clear loss of lubricin fails to present an efficient lubricating surface for the articular cartilage contact and subsequently predisposes articular cartilage surfaces to wear-induced damage. Biomechanical stimulation can modulate lubricin expression $(62,63)$. In the ACL-injured knee, expression of lubricin becomes abnormal with the abnormal articular cartilage contact and pressure distribution $(64,65)$, which may influence integrative cartilage repair (66). Additionally, a number of cytokines and growth factors are involved in the regulation of lubricin expression in the ACL-injured knee. As an anabolic factor, TGF- $\beta 1$ upregulates the synthesis and surface localization of lubricin $(67,68)$, whereas IL-1 or TNF downregulate the production of lubricin $(69,70)$. In an animal model of posttraumatic arthritis, it has been demonstrated that blocking TNF increases lubricin production on the surface of articular cartilage (71). As stated, a severe inflammatory reaction will occur in the ACL-injured knee joint, followed by infiltration of various inflammatory cytokines, such as IL-1 and TNF. Consequently, the normal synthesis of lubricin is affected and lubricin production is significantly reduced with the degenerative processing of cartilage. Therefore, lubricin can be one specific biomarker in synovial fluid.

\section{Conclusions and perspectives}

Biomechanical instability does not appear to be the only cause for the OA changes of the knee joint following the ACL rupture and an alternative etiology by various biochemical factors are postulated to play an important role in the development of posttraumatic OA following ACL injury. In the present study, several important biological factors have been summarized, which participate in the process of articular cartilage damage. Among these factors, inflammatory cytokines were reported to play a significant role in the production of MMPs, which contribute to cartilage PG degradation and collagen destruction. A detailed understanding of these biochemical mediators involved in the matrix degrading process will aid the potential use of specific inhibitors to slow the cartilage degenerative progression and treat the damaged cartilage. Furthermore, several specific biomarkers, including GAGs, CTX-II and lubricin were reviewed. These specific biomarkers can provide effective diagnostic and prognostic tools for identifying subjects with a high risk of progression. Collectively, these results may prompt further studies on the biological prognosis and management of knee joint cartilage damage following ACL rupture.

\section{Acknowledgements}

The present study was subsidized by 973 Project (grant no. 2009CB930000) from the Ministry of Science and Technology of China and Nano Project of Shanghai Municipal Science and Technology Commission (grant no. 1052nm03701).

\section{References}

1. Spindler KP and Wright RW: Clinical practice. Anterior cruciate ligament tear. N Engl J Med 359: 2135-2142, 2008

2. Frobell RB, Roos EM, Roos HP, Ranstam J and Lohmander LS A randomized trial of treatment for acute anterior cruciate ligament tears. N Engl J Med 363: 331-342, 2010. 
3. Brophy RH, Zeltser D, Wright RW and Flanigan D: Anterior cruciate ligament reconstruction and concomitant articular cartilage injury: incidence and treatment. Arthroscopy 26 $112-120,2010$

4. Lohmander LS, Ostenberg A, Englund M and Roos H: High prevalence of knee osteoarthritis, pain, and functional limitations in female soccer players twelve years after anterior cruciate ligament injury. Arthritis Rheum 50: 3145-3152, 2004.

5. Nelson F, Billinghurst RC, Pidoux I, et al: Early post-traumatic osteoarthritis-like changes in human articular cartilage following rupture of the anterior cruciate ligament. Osteoarthritis Cartilage 14: 114-119, 2006.

6. Li G, Moses JM, Papannagari R, Pathare NP, DeFrate LE and Gill TJ: Anterior cruciate ligament deficiency alters the in vivo motion of the tibiofemoral cartilage contact points in both the anteroposterior and mediolateral directions. J Bone Joint Surg Am 88: 1826-1834, 2006.

7. Van de Velde SK, Bingham JT, Hosseini A, et al: Increased tibiofemoral cartilage contact deformation in patients with anterior cruciate ligament deficiency. Arthritis Rheum 60: 3693-3702, 2009.

8. Church S and Keating JF: Reconstruction of the anterior cruciate ligament: timing of surgery and the incidence of meniscal tears and degenerative change. J Bone Joint Surg Br 87: 1639-1642, 2005.

9. Granan LP, Bahr R, Lie SA and Engebretsen L: Timing of anterior cruciate ligament reconstructive surgery and risk of cartilage lesions and meniscal tears: a cohort study based on the Norwegian National Knee Ligament Registry. Am J Sports Med 37: 955-961, 2009.

10. Fajardo M and Di Cesare PE: Disease-modifying therapies for osteoarthritis: current status. Drugs Aging 22: 141-161, 2005.

11. Li H, Hosseini A, Li JS, Gill TJ IV and Li G: Quantitative magnetic resonance imaging (MRI) morphological analysis of knee cartilage in healthy and anterior cruciate ligament-injured knees. Knee Surg Sports Traumatol Arthrosc 20: 1496-1502, 2012.

12. Friel NA and Chu CR: The role of ACL injury in the development of posttraumatic knee osteoarthritis. Clin Sports Med 32: 1-12, 2013.

13. Ferretti A, Conteduca F, De Carli A, Fontana M and Mariani PP Osteoarthritis of the knee after ACL reconstruction. Int Orthop 15: 367-371, 1991

14. Daniel DM, Stone ML, Dobson BE, Fithian DC, Rossman DJ and Kaufman KR: Fate of the ACL-injured patient. A prospective outcome study. Am J Sports Med 22: 632-644, 1994.

15. Li H, Tao H, Hua Y, Chen J, Li Y and Chen S: Quantitative magnetic resonance imaging assessment of cartilage status: a comparison between young men with and without anterior cruciate ligament reconstruction. Arthroscopy 29: 2012-2019, 2013.

16. Oiestad BE, Holm I, Engebretsen L and Risberg MA: The association between radiographic knee osteoarthritis and knee symptoms, function and quality of life 10-15 years after anterior cruciate ligament reconstruction. Br J Sports Med 45: 583-588, 2011.

17. Kraus VB, Birmingham J, Stabler TV, et al: Effects of intraarticular IL1-Ra for acute anterior cruciate ligament knee injury: a randomized controlled pilot trial (NCT00332254). Osteoarthritis Cartilage 20: 271-278, 2012

18. Wei L, Fleming BC, Sun X, et al: Comparison of differential biomarkers of osteoarthritis with and without posttraumatic injury in the Hartley guinea pig model. J Orthop Res 28 : 900-906, 2010.

19. Marks PH and Donaldson ML: Inflammatory cytokine profiles associated with chondral damage in the anterior cruciate ligament-deficient knee. Arthroscopy 21: 1342-1347, 2005.

20. Aigner T, Soeder S and Haag J: IL-1beta and BMPs - interactive players of cartilage matrix degradation and regeneration. Eur Cell Mater 12: 49-56, 2006

21. Turner NA, Warburton P, O'Regan DJ, Ball SG and Porter KE: Modulatory effect of interleukin-1alpha on expression of structural matrix proteins, MMPs and TIMPs in human cardiac myofibroblasts: role of p38 MAP kinase. Matrix Biol 29: 613-620, 2010.

22. Xue R, Yang L, Tang Z, et al: The profile of MMP and TIMP in injured rat ACL. Mol Cell Biomech 7: 115-124, 2010.

23. Chen WP, Tang JL, Bao JP, et al: Effects of diallyl sulphide in chondrocyte and cartilage in experimental osteoarthritis in rabbit. Phytother Res 25: 351-356, 2011.
24. Tang Z, Yang L, Zhang J, et al: Coordinated expression of MMPs and TIMPs in rat knee intra-articular tissues after ACL injury. Connect Tissue Res 50: 315-322, 2009.

25. Ra HJ and Parks WC: Control of matrix metalloproteinase catalytic activity. Matrix Biol 26: 587-596, 2007.

26. Lin PM, Chen CT and Torzilli PA: Increased stromelysin-1 (MMP-3), proteoglycan degradation (3B3- and 7D4) and collagen damage in cyclically load-injured articular cartilage. Osteoarthritis Cartilage 12: 485-496, 2004.

27. Ni GX, Zhan LQ, Gao MQ, Lei L, Zhou YZ and Pan YX: Matrix metalloproteinase-3 inhibitor retards treadmill running-induced cartilage degradation in rats. Arthritis Res Ther 13: R192, 2011.

28. Catterall JB, Stabler TV, Flannery CR and Kraus VB: Changes in serum and synovial fluid biomarkers after acute injury (NCT00332254). Arthritis Res Ther 12: R229, 2010.

29. Hattori S, Sakane M, Mutsuzaki H, Tanaka J, Ochiai N and Nakajima H: Chondrocyte apoptosis and decrease of glycosaminoglycan in cranial cruciate ligament insertion after resection in rabbits. J Vet Med Sci 69: 253-258, 2007.

30. Sakane M, Mutsuzaki H, Hattori S, Nakajima H and Ochiai N: Time dependence of changes of two cartilage layers in anterior cruciate ligament insertion after resection on chondrocyte apoptosis and decrease in glycosaminoglycan. Sports Med Arthrosc Rehabil Ther Technol 1: 27, 2009.

31. Connelly JT, Wilson CG and Levenston ME: Characterization of proteoglycan production and processing by chondrocytes and BMSCs in tissue engineered constructs. Osteoarthritis Cartilage 16: 1092-1100, 2008.

32. Little CB, Hughes CE, Curtis CL, et al: Matrix metalloproteinases are involved in $\mathrm{C}$-terminal and interglobular domain processing of cartilage aggrecan in late stage cartilage degradation. Matrix Biol 21: 271-288, 2002.

33. Troeberg $\mathrm{L}$ and Nagase $\mathrm{H}$ : Proteases involved in cartilage matrix degradation in osteoarthritis. Biochim Biophys Acta 1824: 133-145, 2012.

34. Caterson B, Flannery CR, Hughes CE and Little CB: Mechanisms involved in cartilage proteoglycan catabolism. Matrix Biol 19: 333-344, 2000

35. Sondergaard BC, Schultz N, Madsen SH, Bay-Jensen AC, Kassem M and Karsdal MA: MAPKs are essential upstream signaling pathways in proteolytic cartilage degradation - divergence in pathways leading to aggrecanase and MMP-mediated articular cartilage degradation. Osteoarthritis Cartilage 18: 279-288, 2010.

36. Lorenz H, Wenz W, Ivancic M, Steck E and Richter W: Early and stable upregulation of collagen type II, collagen type I and YKL40 expression levels in cartilage during early experimental osteoarthritis occurs independent of joint location and histological grading. Arthritis Res Ther 7: R156-R165, 2005.

37. Pujol JP, Chadjichristos C, Legendre F, et al: Interleukin-1 and transforming growth factor-beta 1 as crucial factors in osteoarthritic cartilage metabolism. Connect Tissue Res 49: 293-297, 2008.

38. Chadjichristos C, Ghayor C, Kypriotou M, et al: Sp1 and Sp3 transcription factors mediate interleukin-1 beta down-regulation of human type II collagen gene expression in articular chondrocytes. J Biol Chem 278: 39762-39772, 2003

39. Porée B, Kypriotou M, Chadjichristos C, et al: Interleukin-6 (IL-6) and/or soluble IL-6 receptor down-regulation of human type II collagen gene expression in articular chondrocytes requires a decrease of $\mathrm{Sp} 1 . \mathrm{Sp} 3$ ratio and of the binding activity of both factors to the COL2A1 promoter. J Biol Chem 283: 4850-4865, 2008.

40. Mutsuzaki H, Sakane M, Ikeda K, et al: Histological changes and apoptosis of cartilage layer in human anterior cruciate ligament tibial insertion after rupture. Knee Surg Sports Traumatol Arthrosc 15: 602-609, 2007.

41. Mutsuzaki H, Sakane M, Honda K, Ikeda K, Hattori S and Ochiai N: Cell death and cell proliferation in cartilage layers in human anterior cruciate ligament tibial insertions after rupture. Connect Tissue Res 51: 282-288, 2010

42. Nishimuta JF and Levenston ME: Response of cartilage and meniscus tissue explants to in vitro compressive overload. Osteoarthritis Cartilage 20: 422-429, 2012.

43. Xu L, Hayashi D, Roemer FW, Felson DT and Guermazi A: Magnetic resonance imaging of subchondral bone marrow lesions in association with osteoarthritis. Semin Arthritis Rheum 42: 105-118, 2012.

44. Lewis JL, Deloria LB, Oyen-Tiesma M, Thompson RC Jr, Ericson M and Oegema TR Jr: Cell death after cartilage impact occurs around matrix cracks. J Orthop Res 21: 881-887, 2003. 
45. Frobell RB: Change in cartilage thickness, posttraumatic bone marrow lesions, and joint fluid volumes after acute ACL disruption: a two-year prospective MRI study of sixty-one subjects. J Bone Joint Surg Am 93: 1096-1103, 2011.

46. Nakamae A, Engebretsen L, Bahr R, Krosshaug T and Ochi M: Natural history of bone bruises after acute knee injury: clinical outcome and histopathological findings. Knee Surg Sports Traumatol Arthrosc 14: 1252-1258, 2006.

47. Lahm A, Uhl M, Erggelet C, Haberstroh J and Mrosek E: Articular cartilage degeneration after acute subchondral bone damage: an experimental study in dogs with histopathological grading. Acta Orthop Scand 75: 762-767, 2004.

48. Lahm A, Kreuz PC, Oberst M, Haberstroh J, Uhl M and Maier D Subchondral and trabecular bone remodeling in canine experimental osteoarthritis. Arch Orthop Trauma Surg 126: 582-587, 2006.

49. Beekhuizen M, van Osch GJ, Bot AG, et al: Inhibition of oncostatin $\mathrm{M}$ in osteoarthritic synovial fluid enhances GAG production in osteoarthritic cartilage repair. Eur Cell Mater 26: 80-90, 2013.

50. Tsuchida AI, Beekhuizen M, Rutgers M, et al: Interleukin-6 is elevated in synovial fluid of patients with focal cartilage defects and stimulates cartilage matrix production in an in vitro regeneration model. Arthritis Res Ther 14: R262, 2012.

51. Sharif M, Kirwan J, Charni N, Sandell LJ, Whittles C and Garnero P: A 5-yr longitudinal study of type IIA collagen synthesis and total type II collagen degradation in patients with knee osteoarthritis - association with disease progression. Rheumatology (Oxford) 46: 938-943, 2007.

52. Lohmander LS, Atley LM, Pietka TA and Eyre DR: The release of crosslinked peptides from type II collagen into human synovial fluid is increased soon after joint injury and in osteoarthritis. Arthritis Rheum 48: 3130-3139, 2003.

53. Duclos ME, Roualdes O, Cararo R, Rousseau JC, Roger T and Hartmann DJ: Significance of the serum CTX-II level in an osteoarthritis animal model: a 5-month longitudinal study. Osteoarthritis Cartilage 18: 1467-1476, 2010.

54. Freeston JE, Garnero P, Wakefield RJ, Hensor EM, Conaghan PG and Emery P: Urinary type II collagen C-terminal peptide is associated with synovitis and predicts structural bone loss in very early inflammatory arthritis. Ann Rheum Dis 70: 331-333, 2011.

55. Ikegawa S, Sano M, Koshizuka Y and Nakamura Y: Isolation, characterization and mapping of the mouse and human PRG4 (proteoglycan 4) genes. Cytogenet Cell Genet 90: 291-297, 2000

56. Jay GD, Tantravahi U, Britt DE, Barrach HJ and Cha CJ: Homology of lubricin and superficial zone protein (SZP): products of megakaryocyte stimulating factor (MSF) gene expression by human synovial fibroblasts and articular chondrocytes localized to chromosome 1q25. J Orthop Res 19: 677-687, 2001.

57. Liu YJ, Lu SH, Xu B, et al: Hemangiopoietin, a novel human growth factor for the primitive cells of both hematopoietic and endothelial cell lineages. Blood 103: 4449-4456, 2004.
58. Coles JM, Zhang L, Blum JJ, et al: Loss of cartilage structure, stiffness, and frictional properties in mice lacking PRG4. Arthritis Rheum 62: 1666-1674, 2010

59. Schmidt TA, Gastelum NS, Nguyen QT, Schumacher BL and Sah RL: Boundary lubrication of articular cartilage: role of synovial fluid constituents. Arthritis Rheum 56: 882-891, 2007.

60. Rhee DK, Marcelino J, Baker M, et al: The secreted glycoprotein lubricin protects cartilage surfaces and inhibits synovial cell overgrowth. J Clin Invest 115: 622-631, 2005.

61. Elsaid KA, Machan JT, Waller K, Fleming BC and Jay GD: The impact of anterior cruciate ligament injury on lubricin metabolism and the effect of inhibiting tumor necrosis factor alpha on chondroprotection in an animal model. Arthritis Rheum 60: 2997-3006, 2009.

62. Nugent GE, Aneloski NM, Schmidt TA, Schumacher BL, Voegtline MS and Sah RL: Dynamic shear stimulation of bovine cartilage biosynthesis of proteoglycan 4. Arthritis Rheum 54: 1888-1896, 2006.

63. Nugent-Derfus GE, Takara T, O'Neill JK, et al: Continuous passive motion applied to whole joints stimulates chondrocyte biosynthesis of PRG4. Osteoarthritis Cartilage 15: 566-574, 2007.

64. Matyas JR, Atley L, Ionescu M, Eyre DR and Poole AR: Analysis of cartilage biomarkers in the early phases of canine experimental osteoarthritis. Arthritis Rheum 50: 543-552, 2004.

65. Jones AR, Chen S, Chai DH, et al: Modulation of lubricin biosynthesis and tissue surface properties following cartilage mechanical injury. Arthritis Rheum 60: 133-142, 2009.

66. Englert C, McGowan KB, Klein TJ, Giurea A, Schumacher BL and Sah RL: Inhibition of integrative cartilage repair by proteoglycan 4 in synovial fluid. Arthritis Rheum 52: 1091-1099, 2005.

67. Schmidt TA, Gastelum NS, Han EH, Nugent-Derfus GE, Schumacher BL and Sah RL: Differential regulation of proteoglycan 4 metabolism in cartilage by IL-1alpha, IGF-I, and TGF-beta1. Osteoarthritis Cartilage 16: 90-97, 2008.

68. Neu CP, Khalafi A, Komvopoulos K, Schmid TM and Reddi AH: Mechanotransduction of bovine articular cartilage superficial zone protein by transforming growth factor beta signaling. Arthritis Rheum 56: 3706-3714, 2007.

69. Gleghorn JP, Jones AR, Flannery CR and Bonassar LJ: Alteration of articular cartilage frictional properties by transforming growth factor beta, interleukin-1beta, and oncostatin M. Arthritis Rheum 60: 440-449, 2009.

70. Elsaid KA, Fleming BC, Oksendahl HL, et al: Decreased lubricin concentrations and markers of joint inflammation in the synovial fluid of patients with anterior cruciate ligament injury. Arthritis Rheum 58: 1707-1715, 2008.

71. Elsaid KA, Jay GD, Warman ML, Rhee DK and Chichester CO: Association of articular cartilage degradation and loss of boundary-lubricating ability of synovial fluid following injury and inflammatory arthritis. Arthritis Rheum 52: 1746-1755, 2005 . 